\title{
A review of the use of exemestane in early breast cancer
}

\author{
Andrew Robinson \\ Northern Ontario School of Medicine, \\ Regional Cancer Program of Sudbury \\ Regional Hospital, Sudbury, Ontario, \\ Canada
}

\begin{abstract}
Exemestane is a third-generation aromatase inhibitor, which has proven to be a useful drug in the treatment of early stage breast cancer. Several clinical trials have been performed or are currently underway using exemestane as adjuvant therapy in postmenopausal women, which will be the indication reviewed here. A relative reduction in risk of breast cancer recurrence or death of $24 \%$ has been shown with exemestane compared with tamoxifen when given after 2 to 3 years of tamoxifen. This corresponded to a $3.3 \%$ absolute reduction in recurrence or death at the end of 5 years, for a number needed to treat of 30 . The main use of exemestane in the adjuvant setting is as an alternative to tamoxifen, and toxicities are discussed in relation to tamoxifen toxicities. In general, patients receiving exemestane experience less hot flashes and more arthralgias in comparison to tamoxifen, while there is also a reduction in venous thromboembolic events and vaginal bleeding. Patients on exemestane as a group do not appear to have a significantly changed quality of life in comparison to tamoxifen, while having a statistically significant benefit in preventing breast cancer recurrence.
\end{abstract}

Keywords: breast cancer, exemestane, adjuvant

\section{Background}

Exemestane (6-methylenandrosta-1,4-diene-3,17-dione) is a third-generation irreversible aromatase inhibitor currently in clinical use for the treatment of both advanced and early breast cancer. This review will focus on its role in the adjuvant treatment of breast cancer.

Breast cancer is the most prevalent cancer in women worldwide, and responsible for the greatest number of deaths. ${ }^{1}$ Almost half a million women will die of breast cancer each year. In the developed countries, approximately $70 \%$ of breast cancers express the estrogen receptor, ${ }^{2}$ and hormonal manipulation in terms of blocking or inactivating the receptor, or reducing the amount of estrogen available, has been an important advance in the treatment of these women. ${ }^{3}$ In the late $1800 \mathrm{~s}$, case reports of oophorectomy benefiting patients with breast cancer ${ }^{4}$ and the subsequent application in the 1950s of oophorectomy and adrenalectomy for patients with advanced breast cancer, set the foundation for the critical role that hormonal manipulation currently plays in the treatment of breast cancer. ${ }^{5}$

In the setting of adjuvant treatment of early breast cancer, anti-estrogen therapy has been shown to reduce the risk of breast cancer recurrence and death. The standard for the adjuvant hormonal treatment for early stage breast cancer has been tamoxifen citrate (tamoxifen), a selective estrogen receptor modulator (SERM). ${ }^{3}$ As a SERM, tamoxifen exerts both antagonistic and agonistic effects on the estrogen receptor, depending on the tissue, the hormonal milieu, and possibly depending on what other receptor pathways and coregulators are activated. Tamoxifen has been well studied in over 20,000 women, and reduces the risk of death by a relative amount of $22 \%$, and the risk of recurrence by $42 \% .^{3}$ 
Despite the clinical success of tamoxifen, attempts have been made to find alternate pharmacologic strategies with a greater therapeutic index in the adjuvant setting. Exemestane has been shown to modestly improve efficacy in terms of breast cancer recurrence, and to modestly reduce adverse events such as endometrial cancer, and venous thromboembolism. ${ }^{6}$ This review will focus on the pharmacologic and clinical aspects of exemestane as adjuvant treatment in early breast cancer.

\section{Pharmacologic properties}

Exemestane is an irreversible, highly specific, inhibitor of the cytochrome p450 enzyme, aromatase. Aromatase is an enzyme found in many tissues, including adipose, skin, adrenal, and breast tissue (and in breast tumor tissue) and is a catalyst for the final step of estrogen synthesis, the NADPH-dependent conversion of androstenedione to estrogens. ${ }^{7}$ The aromatase enzyme is located intracellularly, in the endoplasmic reticulum. Because current aromatase inhibitors target this final step of estrogen synthesis, little effect on other adrenal hormones occurs. Exemestane is a molecule with a steroidal structure similar to androstenedione, and it acts as a false substrate for aromatase. Once it is recognized by aromatase, it is converted to an intermediate which binds irreversibly to the aromatase enzyme, thus inactivating it. For estrogen synthesis to begin again, new aromatase must be produced.

Exemestane is highly bioavailable, with $42 \%$ oral availability. The plasma half-life of exemestane is $27 \mathrm{~h}$, and the time to steady state concentration is 7 days or less. ${ }^{8}$ Exemestane is metabolized by the CYP3A4 system, although clinically significant interactions with CYP3A4 inhibitors such as ketoconazole have not been demonstrated. ${ }^{9} \mathrm{CYP} 3 \mathrm{~A} 4$ inducers, such as phenytoin, may lower plasma concentrations of exemestane, although again the clinical significance is unclear. Excretion occurs via both the hepatic and renal pathways, however dose adjustments are not necessary.

Pharmacodynamically, exemestane exerts its effects by irreversibly inactivating the aromatase enzyme. Plasma estrogen levels drop by $90 \%$ in postmenopausal women, with systemic drops in estrone of $95 \%$ and estradiol of $85 \%$, as well as estriol, after a single dose of $25 \mathrm{mg}$. Estrogen levels remain suppressed for 4 days, despite the half-life of exemestane being considerably shorter. ${ }^{8}$ This is due to the fact that exemestane irreversibly inhibits the aromatase enzyme. Exemestane also inhibits intratumoral levels of estrogen, although the clinical significance of this is not clear. ${ }^{10}$

Although not currently standard of care in premenopausal patients, it is important to note that when exemestane is given in combination therapy with the LHRH agonist triptorelin in premenopausal patients, a significant drop of estrogen levels in premenopausal patients results, in comparison to triptorelin alone. ${ }^{11}$

\section{Clinical effectiveness in early breast cancer}

Exemestane has been studied in the adjuvant setting in postmenopausal patients in a series of trials that are either completed or ongoing. In the postmenopausal adjuvant setting, the landmark International Exemestane Study (IES) investigated the use of exemestane for 2 to 3 years following the use of tamoxifen for 2 to 3 years. ${ }^{12}$ The Tamoxifen or Exemestane Adjuvant Multicentre study (TEAM) initially was designed to investigate 5 years of tamoxifen versus 5 years of exemestane, but after the results of the IES study were known it was amended to investigate the option of 5 years of exemestane versus 5 years of a tamoxifen followed by exemestane sequence (ie, similar to the experimental arm of the IES study). ${ }^{13}$ The National Cancer Institute of Canada (NCIC) is currently running the MA-27 study, which is a direct comparison of the steroidal aromatase inhibitor exemestane with the non-steroidal aromatase inhibitor anastrozole. This will be the first study to look at whether there are any significant differences between the third generation aromatase inhibitors. The NSABP B-33 study examined the question of whether exemestane was beneficial after 5 years of tamoxifen treatment, and was the only trial with a placebo control. ${ }^{14}$ This study was stopped early due to the results of other aromatase inhibitors showing benefit in a similar setting.

In the premenopausal setting, exemestane is being investigated in combination with ovarian suppression in the Suppression of Ovarian Function Trial (SOFT study), which is randomizing patients to tamoxifen alone, or to ovarian suppression with tamoxifen, or to ovarian suppression with exemestane. The Tamoxifen or Exemestane Trial (TEXT) is comparing tamoxifen with exemestane in patients with ovarian suppression with a LHRH agonist - triptorelin. ${ }^{15}$

In the neoadjuvant setting, exemestane has been studied in both randomized controlled trials and in many phase 2 settings. While these trials have raised some interesting biological questions, and neoadjuvant exemestane may be an option in locally advanced elderly patients, ${ }^{16-18}$ the clinical relevance of neoadjuvant aromatase inhibition in early breast cancer is minimal.

\section{The IES study}

The IES study, which was a landmark study, randomized patients who had received 2 to 3 years of tamoxifen for adjuvant 
breast cancer treatment to either continue tamoxifen or switch to exemestane for the duration of 5 years. 4724 patients were enrolled on the study, all of whom were postmenopausal. As of the most recent published analysis, median follow-up was 55.7 months. Disease-free survival, defined in this study as a composite endpoint of death from any cause, new breast cancer, or recurrence of breast cancer at any site, was statistically significantly improved, with a relative improvement of 24\% (hazard ratio [HR] 0.76). Overall survival approached statistical significance, and when the subset of patients with known estrogen receptor negativity was excluded, risk of death was $17 \%$ lower in the exemestane group, with an HR ratio of 0.83 , and $95 \%$ confidence intervals (CI) just touching unity $(0.69-1.00){ }^{12}$

The patients in the IES study represented patients who had already received at least 2 years of tamoxifen without recurrence, and had very strict definitions of postmenopausal status. Patients had to have no menses for 2 years and be over age 55, or have had no menses from 1 year before cancer diagnosis. In other words, patients in their 40s who became amennorheic with chemotherapy would not have been eligible for this study. Approximately half of the patients were node negative, half had primary tumors less than $2.0 \mathrm{~cm}$ (ie, T1), and two thirds were greater than 60 years old. The absolute benefit in the trial population group in comparison to tamoxifen was $3.3 \%$, meaning that in a similar selection of patients, roughly 30 patients would need to be treated with exemestane to prevent one recurrence or death at the end of 5 years. The absolute difference in death at the end of treatment was $0.72 \%$ in patients without estrogen receptor positivity, for a number needed to treat of roughly 138 patients to prevent 1 death.

\section{The NSABP B-33 study}

The NSABP B-33 study enrolled patients who had completed 5 years of tamoxifen. Patients were randomized to placebo or exemestane for a further 5 years, with a planned sample size of 3000 patients. The trial was terminated early due to the results of the NCIC MA-17 study which enrolled a similar group of patients to a trial comparing placebo and the non-steroidal aromatase inhibitor letrozole. In that study, letrozole was associated with a statistically significant improvement of disease-free survival, with a $4.6 \%$ absolute improvement in disease-free survival at 4 years $(\mathrm{p}<0.001)$, and a subset analysis of overall survival showed benefit in node-positive patients. Thus, it was elected to terminate NSABP-B33 early, with an accrual of 1598 patients.
The results of this study were not statistically positive, possibly due to the attenuated accrual and thus lack of statistical power. The HR for overall survival was 1.20 , with CI from 0.57 to 2.52 . This study will be important to add to future meta-analyses of the utility of extended adjuvant therapy. In terms of disease-free survival, a $2 \%$ absolute reduction in disease free survival was seen for the exemestane arm, although this again did not meet statistical significance due to the lack of power $(p=0.07)$. A third endpoint of the trial, recurrence-free survival, did show a statistically significant advantage of exemestane over placebo, with $94 \%$ of patients being breast cancer relapse free at 4 years, with $96 \%$ in the exemestane arm being breast cancer relapse free. The majority of this benefit was due to reduced local recurrence and contralateral breast cancer. The number of deaths in trial was very few, with only 13 deaths in the placebo arm and 16 deaths in the exemestane arm. Whether exemestane is a reasonable option for patients who cannot tolerate or obtain letrozole in the extended adjuvant setting is controversial, and depends on how one weighs the benefits of breast cancer recurrence prevention with the lack of benefit thus far in terms of overall survival. ${ }^{14}$

In terms of trials using 5 years of exemestane as the initial adjuvant treatment, there are as yet no completed and reported clinical trials using this strategy. The MA27 study, compares exemestane with anastrozole for 5 years, and the TEAM study compares the 'sequence strategy' of tamoxifen and exemestane with 5 years of exemestane straight. Both of these trials will be looking at 5 years of exemestane as the experimental treatment, with different comparison arms. Currently, 5 years of upfront exemestane treatment should not be considered an appropriate therapy based on the completed clinical trials.

\section{Pre- and peri-menopausal patients}

In the pre-menopausal setting, the majority of estrogen production occurs in the ovaries, and inhibition of adrenal and peripheral aromatase as a sole-modality of treatment is does not lower systemic estrogen levels to a significant amount. Thus, in the pre-menopausal setting, exemestane in addition to ovarian suppression is being evaluated as an adjuvant strategy. The SOFT study - the Suppression of Ovarian Function study - is a three armed study of various anti-estrogen strategies in pre-menopausal patients, including tamoxifen alone, ovarian suppression plus tamoxifen, or ovarian suppression plus exemestane. The TEXT study is simply randomizing patients between ovarian suppression plus tamoxifen versus ovarian suppression plus exemestane. 
Results from these two trials are anticipated, but currently exemestane is not an evidence based option for the adjuvant treatment of premenopausal breast cancer.

In addition, premenopausal patients who become amennorheic after chemotherapy and are treated with exemestane, may have ovarian function return. An audit from the Royal Marsden Hospital of patients over the age of 40 who had chemotherapy induced amenorrhea and subsequently received an aromatase inhibitor showed that $27 \%$ of patients recovered ovarian function, which was higher than anticipated. A significant proportion of these had received tamoxifen for 1 to 3 years prior to switching to an aromatase inhibitor. Aromatase inhibitors such as exemestane should only be used with extreme caution in patients under 50 with chemotherapy induced amenorrhea, if at all. ${ }^{19}$

\section{Toxicities}

In assessing the toxicities associated with exemestane, it is important to compare the toxicities with those of tamoxifen, given that the major use of exemestane is in a substitution for tamoxifen at the 2- to 3-year mark. The NSABP-B33 trial comparing exemestane to placebo gives some idea as to what toxicities are truly due to exemestane, as will the current exemestane prevention study (ExCel) in patients at a "high" risk of developing breast cancer (http://www. excelstudy.com/).

\section{Life-threatening side-effects} Thromboembolic disease/cardiovascular disease

Exemestane has a clear advantage over tamoxifen in the risk of venous thromboembolic disease. Tamoxifen, a partial estrogen agonist, does increase the risk of thromboembolism slightly over baseline. ${ }^{20}$ In the IES study, venous thromboembolic disease, defined as deep venous thrombosis or pulmonary embolism, occurred in $2.3 \%$ of patients in the tamoxifen arm and $1.2 \%$ of patients in the exemestane arm. This difference was statistically significant $(p=0.004)$. No patients in either arm had documented death due to venous thromboembolic disease. ${ }^{12}$

In terms of cardiovascular events, $16.5 \%$ of patients in the exemestane arm and 15.0 percent of patients in the tamoxifen arm had a cardiovascular event. This includes $8.0 \%$ of people and $6.9 \%$ of people having ischemic heart event respectively. These differences did not reach statistical significance $\left(\mathrm{p}=0.16\right.$ and $\mathrm{p}=0.17$ respectively).${ }^{12}$ It can be concluded from this study, that at least as far as 5 years from switching to exemestane, there was no statistically significant detriment in terms of cardiovascular events by using the steroidal aromatase inactivator exemestane.

It should be noted that if a $1.1 \%$ absolute increase in thromboembolic events is considered within the realm of clinical significance, then an absolute increase of $1.1 \%$ increase in ischemic heart events would also be expected to be within the realm of clinical significance. However, given the rare nature of venous thromboembolic events in comparison to ischemic heart events in the population, the chance of determining a statistically significant difference with the same absolute difference is much higher for rare events.

\section{Bone fractures}

Just as thromboembolic and cardiovascular events range from relatively easily treated events with a low likelihood of mortality to more serious events such as life threatening pulmonary embolism, so too do bone fractures range in severity from fractures associated with some morbidity, such as Colles' fractures and vertebral fractures, to fractures associated with a high mortality, such as hip fractures in the elderly. ${ }^{21}$ Tamoxifen may exert some bone protective effects, as measured by an increase in bone-density. In clinical trials compared with placebo, tamoxifen was shown to possibly reduce the incidence of osteoporotic fractures by $19 \%$, although this did not reach statistical significance. ${ }^{22}$ A recent population based study also showed that tamoxifen use was associated with a reduced rate of fractures. ${ }^{23}$ There is controversy over how bone protective tamoxifen is, however, and how long the effect lasts, as increases in bone density do not always equate with fracture prevention..$^{24}$ Given the similar benefit for osteoporotic fractures seen with the selective estrogen receptor modulator raloxifene, as well as various observational studies with tamoxifen, it is likely that there is probably a decrease in fracture risk with tamoxifen.

Aromatase inhibition with exemestane, which further lowers postmenopausal estrogen levels, has the concern of further exacerbating the natural bone-loss of menopause. Information from the IES and TEAM studies will not tell us whether exemestane has a detrimental effect on bone structure or bone density scores, but will tell us if the effect is different from tamoxifen. Data from the NSABP-B33 study, and the exemestane prevention studies, will tell us what the risk of bone problems is in relation to placebo, and data from the MA27 study will tell us how the risk of bone metabolism issues with a steroidal aromatase inactivator such as exemestane compares with the non-steroidal aromatase inhibitor exemestane. 
In the IES study, at a median follow-up in of 58 months, $162(7 \%)$ and $115(5 \%)$ patients in the exemestane and tamoxifen groups, respectively, had fractures (odds ratio 1.45 [1.13-1.87]; $p=0.003)$. In other words, 50 patients would need to be treated with exemestane instead of tamoxifen in order to see one additional fracture (number needed to harm $=50$ ). This number must be compared to the number needed to treat of 33 to prevent one death or breast cancer recurrence. In terms of bone density as measured by DEXA scans, and markers of bone turnover such as n-telopeptides, there was statistically significant loss of bone density and increase in biochemical markers in the first year of switching. The amount of bone density loss decreased from the first to second year. ${ }^{25}$

In the NSABP B-33 study, very few fractures had occurred, with 28 fractures in the exemestane group and 20 in the placebo group $(3.5 \%$ vs $2.6 \% \mathrm{p}=\mathrm{NS})$. Further data on incidences of osteopenia, or other bone-related abnormalities, are awaited. As this study was unblinded earlier than anticipated, and $44 \%$ of patients who had been randomized to placebo subsequently crossed over to receive exemestane, the fracture rates will not be indicative of 5 years of exemestane compared to 5 years of placebo. ${ }^{14}$

Bone mineral density abnormalities and fractures are a recognized complication of aromatase inhibitor therapy, and may occur after treatment has been discontinued. Appropriate attention to bone density monitoring, and the initiation of bone health strategies are advised. In addition to antiresorptive pharmacologic therapies such as bisphosphonates, other aspects of osteoporosis fracture prevention, such as fall prevention, avoidance of CNS depressant drugs such as benzodiazepines in persons at risk of fracture, encouragement of weight bearing exercise, and other general bone health strategies are advised. ${ }^{26}$

\section{Lipid side-effects}

Although cardiovascular events in the first 5 years were not statistically significantly adversely effected by exemestane in relation to tamoxifen, there may be an effect on lipid profiles. In the TEAM study comparing exemestane with tamoxifen, a substudy was done looking at the effects on lipid profiles of the two drugs. 176 patients were included in this study, with lipid profiles including HDL, LDL, triglycerides, and total cholesterol, measured at different time points. There was a decrease in total cholesterol in the tamoxifen group by $9.9 \%$, and a decrease in the exemestane group of $1.9 \%$ $(p=0.039)$ at 12 months. Triglyceride levels were higher in the tamoxifen arm, while LDL levels were higher in the exemestane arm at the 3- and 6-month mark. ${ }^{27}$ It is unclear whether such minor differences in lipid profiles will result in any significant clinical outcomes at later time periods.

\section{Gynecologic side-effects}

The association of tamoxifen with endometrial cancer has been well established. In terms of absolute numbers, tamoxifen therapy increases the risk of endometrial cancer by 1 case per 1000 women treated with tamoxifen per year. ${ }^{28}$ Although many of these cancers are caught early, the risk of death from endometrial cancer does exist. In the IES study, there was no significant difference in endometrial cancers between the exemestane and tamoxifen arms, and risks were very low. The tamoxifen treated patients did have a higher rate of vaginal bleeding, a higher rate of vaginal discharge, and a higher rate of dilatation and curettage. ${ }^{12}$ In the IES Quality of Life substudy, there was less vaginal discharge associated with exemestane, with no significant increase in vaginal dryness. ${ }^{29}$ In contrast, in the TEAM study, exemestane patients, in contrast, had significantly higher rates of vaginal dryness in comparison to tamoxifen treated patients. ${ }^{30}$ In addition, atrophic vaginitis has been reported to be as common as $20 \%$ in patients receiving aromatase inhibitors. ${ }^{31}$ The management of vaginal dryness in patients on aromatase inhibitors generally begins with topical non-hormonal lubricants. Hormonal based vaginal suppositories and creams, may increase systemic estrogen levels in these patients, which is an effect not generally wanted in breast cancer. ${ }^{32}$ It is unclear at this time whether lower dose topical estrogens, such as Estring ${ }^{\circledR}$, are entirely safe, or whether it is better to switch these patients back to tamoxifen. Aromatase inhibitors, including exemestane, lead to an increase in reported dyspareunia, and may lead to decreased sexual satisfaction. ${ }^{33}$

\section{Non-gynecological menopausal-type side-effects}

Estrogen deprivation with aromatase inhibitors has other side-effects apart from the gynecological tract and bone metabolism. Hot flashes are generally less with exemestane than with tamoxifen, and tend to peak at around three months. In contrast, the incidence of decreased libido is slightly greater with exemestane (possibly in part due to the dyspareunia secondary to vaginal dryness), and difficulty sleeping occurs more frequently with exemestane in comparison to tamoxifen. Effects on cognitive abilities do not appear to be significantly different. ${ }^{30}$

\section{Arthralgias and myalgias}

One of the most frequent side-effects experienced by patients on aromatase inhibitors, including exemestane, is 
that of muscle and joint aches. In the IES study, arthralgia, carpal tunnel syndrome, joint stiffness, and paresthesia were reported in more patients in the exemestane arm than in the tamoxifen arm. In terms of arthralgia, $18.6 \%$ of patients on the exemestane arm reported arthralgia, compared with $11.8 \%$ in the tamoxifen arm. Musculoskeletal pain also occurred more frequently on the exemestane arm, with $21.0 \%$ of patients in the exemestane arm and $16.1 \%$ of patients in the tamoxifen arm experiencing this pain. ${ }^{12}$ Whether these relatively low numbers are representative of clinical practice in the "real world" setting has been a matter of debate, as reported incidences of musculoskeletal complaints in many series significantly exceeds $21 \%$. A recent survey of patients taking aromatase inhibitors for early stage breast cancer showed that the reported incidence of joint pain or stiffness was greater than $40 \% .{ }^{34}$ Evidence from more recent studies, such as the MA27 study, may give a better idea of the incidence, given the increased awareness of this phenomenom with clinical experience. The issue of the true incidence of aromatase inhibitor joint toxicity is difficult to evaluate from clinical trials, given the inadequacy of the commonly used observer related grading systems used to assess these long term subjective issues.

The mechanism of the joint and muscle complaints is not fully understood currently. Certainly, the incidence of carpal tunnel syndrome in the IES study of $2.8 \%$, compared with the incidence of $0.3 \%$ in the tamoxifen arm, suggests a certain biological phenomenom. In addition, MRI studies have shown increased tenosynovial thickening in patients on aromatase inhibitors, and objective measurement of joint function, such as grip strength is impaired in patients on aromatase inhibitors. ${ }^{35}$

Management of exemestane associated arthralgias and myalgias includes conventional maneuvers such as exercise and analgesia. ${ }^{36}$ If symptoms persist, one can decide based on the baseline risk of death from cancer in the patient whether continuing with exemestane and adding anti-inflammatory medicines or acetaminophen is warranted, or whether switching to different aromatase inhibitors and possibly back to tamoxifen would be reasonable. Certainly, the balance between the risks of a small increase in breast cancer recurrence in comparison with the risks of polypharmacy, long term issues with anti-inflammatory medicines, for example, must be weighed on an individual level. ${ }^{37}$ In addition, it is unclear whether the symptoms of arthralgias are indicative of true joint damage, and whether this symptom should be masked. Estrogen replacement therapy, in some studies, has been shown to lower the incidence of osteoarthritis in postmenopausal women, ${ }^{38}$ and it is plausible that further lowering of estrogen beyond that of a postmenopausal woman will exacerbate osteoarthritis. While controlling symptoms with analgesia may increase the compliance associated with aromatase inhibitors such as exemestane, it is unclear if the underlying joint damage is still occurring in the absence of symptoms. Again, the known benefits in terms of breast cancer reduction must be weighed in the individual patient against the known current toxicities, and possible future toxicities such as need for joint replacement.

\section{Side-effects and compliance}

In the IES quality of life substudy, 582 patients were enrolled and completed validated questionnaires evaluating quality of life. At the 6-month mark, the composite QOL indicator - the trial outcome index (TOI) was statistically significantly lower among patients receiving exemestane. ${ }^{29}$ However, by the 12-month mark the TOIs return to similar levels. This difference, although statistically significant, is below the level accepted as clinically significant. Importantly from this study, it would appear that quality of life may worsen and then improve on exemestane.

In the IES trial, 2352 patients were randomized to exemestane, and 513 of these patients did not finish treatment. While 133 patients discontinued treatment due to death or disease recurrence, a further 321 , or $14 \%$, discontinued due to adverse events or patient refusal. ${ }^{12}$ Both a chart review of patients receiving adjuvant aromatase inhibitor therapy in Ottawa, Canada, and a large database analysis from the United States, have shown discontinuation or non-adherence rates in the $20 \%$ range. Reasons for discontinuing drug vary, and are comparable to discontinuation rates with tamoxifen. Reasons for discontinuation may be complex, and include physical reasons such as arthralgias, myalgias, hot flashes, vaginal dryness, sleep disturbances. Management of these side-effects, reassurance of the transient nature of some of these effects, and acknowledgement of these side-effects are all strategies to reduce the discontinuation of this drug. ${ }^{36}$ Because other aromatase inhibitors, and other anti-estrogen strategies such as tamoxifen also have proven efficacy in breast cancer, changing treatments is an option in the face of intolerable, or difficult to manage side-effects.

A recent study by Thomas et al examined switching to letrozole or exemestane in patients who had significant hot flashes with tamoxifen. ${ }^{39}$ This study included 80 women with greater than NCI grade 3 hot flashes, or had an average of 14 hot flashes per week, who switched to exemestane. At 3 months after switching, $68 \%$ of the patients preferred exemestane over 
tamoxifen, while $38 \%$ of patients preferred tamoxifen. A minority of patients, $10 \%$, switched back to tamoxifen due to intolerable side-effects. This study confirms the beneficial effects of exemestane on hot flash symptomology, and the detrimental effects of arthralgias. It also confirms that for patients intolerant of tamoxifen, switching to exemestane may be beneficial for their quality of life for some patients. Importantly, almost all patients (99\%) appreciated the opportunity to experience both drugs, and thus to decide for themselves which drug fits best into their life-style.

\section{Summary}

Exemestane has a proven benefit in the treatment of early stage breast cancer, and currently is used in postmenopausal patients, usually as a "switch" strategy after tamoxifen. The use of exemestane is undergoing active clinical trials in other scenarios, such as in premenopausal patients in conjunction with ovarian ablation, or in postmenopausal patients who have not received 2 years of tamoxifen, and in the prevention of breast cancer in high risk women.

While patients need to be counseled regarding the improved breast cancer outcomes and overall survival outcomes related to the usage of exemestane, they also need to be aware of the potential reduction in tamoxifen related toxicities, and in the potential increase in aromatase inhibitor related toxicities. Management of the side-effects and toxicities associated with exemestane involves attention to the bone health, joint health, as well as the vaginal and sexual health of postmenopausal patients with breast cancer.

\section{Disclosures}

The author has no conflicts of interest to declare.

\section{References}

1. Parkin D, Bray F, Ferlay J, Pisani P. Global Cancer Statistics, 2002. CA Cancer J Clin. 2005;55:74-108.

2. Joslyn SA. Hormone receptors in breast cancer: racial differences in distribution and survival. Breast Cancer Res Treat. 2002;73:45-59.

3. Early Breast Cancer Trialists' Collaborative Group. Effects of chemotherapy and hormonal therapy for early breast cancer on recurrence and 15-year survival: an overview of the randomised trials. Lancet. 2005;365: 1687-1717.

4. Beatson GW. On the treatment of inoperable cases of carcinoma of the mamma: suggestions for a new method of treatment with illustrative cases. Lancet. 1896;148(3802):104-107.

5. Huggins C, Dao TL. Adrenalectomy and oophorectomy in treatment of advanced carcinoma of the breast. JAMA. 1953;151:1388-1394.

6. Coombes RC, Hall E, Gibson LJ, et al. A randomized trial of exemestane after two to three years of tamoxifen therapy in post-menopausal women with primary breast cancer. N Engl J Med. 2004;350:1081-1092.

7. Zilembo N, Noberasco C, Bajetta E, Martinetti A, Mariani L, Orefici S. Endocrinological and clinical evaluation of exemestane, a new steroidal aromatase inhibitor. Br J Cancer. 1995;72:1007-1012.
8. Spinelli R, Jannuzzo MG, Poggesi I, Frevola L, Broutin F, Cicioni P Pharmacokinetics (PK) of Aromasin (Exemestane, EXE) after single and repeated doses in healthy postmenopausal volunteers (HPV). Eur J Cancer. 1999;35 suppl 4:S295.

9. Anonymous. Exemestane for advanced breast cancer. Med Lett Drugs Ther. 2000;42:35-36.

10. Dixon JM, Grattage L, Renshaw L, Miller WR. Exemestane as neoadjuvant treatment for locally advanced breast cancer: endocrinologic and clinical endpoints. Breast Cancer Res Treat. 2000;64:53.

11. Jannuzzo M, Di Salle E, Spinelli R, et al. Estrogen suppression in premenopausal women following 8 weeks of treatment with exemestane and triptorelin versus triptorelin alone. Breast Cancer Res Treat. 2008. [Epub ahead of print].

12. Coombes RC, Kilburn LS, Snowdon CF, et al. Survival and safety of exemestane versus tamoxifen after 2-3 years' tamoxifen treatment (Intergroup Exemestane Study): a randomised controlled trial. Lancet. 2007;369(9561):559.

13. Jones, SE. Exemestane as adjuvant treatment of early breast cancer: intergroup exemestane study/tamoxifen exemestane adjuvant multicenter trials. Clin Breast Cancer. 2006;6 suppl 2:S41-S44.

14. Mamounas EP, Jeong J-H, Wickerham DL, et al. Benefit from exemestane as extended adjuvant therapy following five years of adjuvant tamoxifen: Intent-to-treat analysis of the National Surgical Adjuvant Breast and Bowel Project B-33 Trial. J Clin Oncol. 2008;26:1965-1971.

15. Dellapasqua S, Colleoni M, Gelber R, Goldhirsh A. Adjuvant endocrine therapy for premenopausal women with early breast cancer. $J$ Clin Oncol. 2005;26:1736-1750.

16. Takei H, Suemasu K, Inoue K, et al. Multicenter phase II trial of neoadjuvant exemestane for postmenopausal patients with hormone receptorpositive, operable breast cancer: Saitama Breast Cancer Clinical Study Group (SBCCSG-03). Breast Cancer Res Treat. 2008;107:87-94.

17. Tubiani Hulin M, Becette V, Bieche I, et al. Exemestane as neoadjuvant hormonotherapy for locally advanced breast cancer: results of a phase II trial. Anticancer Res. 2007;27:2689-2696.

18. Mlineritsch B, Tausch C, Singer C, et al. Exemestane as primary systemic treatment for hormone receptor positive post-menopausal breast cancer patients: a phase II trial of the Austrian Breast and Colorectal Cancer Study Group (ABCSG-17). Breast Cancer Res Treat. 2007. [Epub ahead of print].

19. Smith IE, Dowsett M, Yap YS, et al. Adjuvant aromatase inhibitors for early breast cancer after chemotherapy-induced amenorrhoea: caution and suggested guidelines. J Clin Oncol. 2006;24:2444-2447.

20. Deitcher SR, Gomes SP. The risk of venous thromboembolic disease associated with adjuvant hormone therapy for breast carcinoma: a systematic review. Cancer. 2004;101(3):439-449.

21. Cummings SR, Melton LJ. Epidemiology and outcomes of osteoporotic fractures. Lancet. 2002;359(9319):1761-1767.

22. Fisher B, Constantino JP, Wickerham DL, et al. Tamoxifen for prevention of breast cancer: report of the National Surgical Adjuvant Breast and Bowel Project P-1 Study. J Natl Cancer Inst. 1998;90(18):1371-1388.

23. Cooke AL, Metge C, Lix L, Prior HJ, Leslie WD. Tamoxifen use and osteoporotic fracture risk - A population based analysis. J Clin Oncol. 2008; Epub October 6.

24. Ding H, Field TS. Bone health in postmenopausal women with early breast cancer: how protective is tamoxifen? Cancer Treat Rev. 2007;33(6):506-513.

25. Coleman RE, Banks LM, Girgis IS, et al. Skeletal effects of exemestane on bone-mineral density, bone biomarkers, and fracture incidence in postmenopausal women with early breast cancer participating in the Intergroup Exemestane Study (IES): a randomised controlled study. Lancet Oncol. 2007;8(2):119-127.

26. Kessel B. Hip fracture prevention in postmenopausal women. Obstet Gynecol Surv. 2004;59(6):446-455.

27. Markopolous C, Polychronis A, Zobolas V, et al. The effect of exemestane on the lipidemic profile of postmenopausal early breast cancer patients: preliminary results of the TEAM Greek sub-study. Breast Cancer Res Treat. 2005;93(1):61-66. 
28. Rutqvist LE, Johansson H, et al. Long-term follow-up of the randomized Stockholm trial on adjuvant tamoxifen among postmenopausal patients with early stage breast cancer. Acta Oncol. 2007;46(2):133-145.

29. Fallowfield L, Bliss J, Porter L, et al. Quality of life in the Intergroup Exemestane Study: a randomized trial of exemestane versus continued tamoxifen after 2 to 3 years of tamoxifen in postmenopausal women with primary breast cancer. J Clin Oncol. 2006;24:910-917.

30. Jones SE, Cantrell J, Vukelja S, et al. Comparison of menopausal symptoms during the first year of adjuvant therapy with either exemestane or tamoxifen in early breast cancer: report of a Tamoxifen Exemestane Adjuvant Multicenter trial substudy. J Clin Oncol. 2007;25(30):4765-4771.

31. Derzko C, Elliott S, Lam W. Management of sexual dysfunction in postmenopausal breast cancer patients taking adjuvant aromatase inhibitor therapy. Curr Oncol. 2007;14 Suppl 1:S20-S40.

32. Kendall A, Dowsett M, Folkerd E, Smith I. Caution: Vaginal estradiol appears to be contraindicated in postmenopausal women on adjuvant aromatase inhibitors. Ann Oncol. 2006;17(4):584-587.

33. Mok K, Juraskova I, Friedlander M. The impact of aromatase inhibitors on sexual functioning: Current knowledge and future research directions. Breast. 2008 May 12 [epub ahead of print].
34. Presant CA, Bosserman L, Young T, et al. Aromatase inhibitor-associated arthralgia and/ or bone pain: frequency and characterization in non-clinical trial patients. Clin Breast Cancer. 2007;7(10):775-778.

35. Morales L, Pans S, Verschueren K, et al. Prospective study to assess short-term intra-articular and tenosynovial changes in the aromatase inhibitor-associated arthralgia syndrome. J Clin Oncol. 2008 May 12 [Epub ahead of print].

36. Cella D, Fallowfield LJ. Recognition and management of treatmentrelated side effects for breast cancer patients receiving adjuvant endocrine therapy. Breast Cancer Res Treat. 2008;107(2):167-180.

37. Francheschi M, Scarcelli M, Niro V, et al. Prevalence, clinical features and avoidability of adverse drug reactions as cause of admission to a geriatric unit: a prospective study of 1756 patients. Drug Saf. 2008;31(6):545-556.

38. Cirillo DJ, Wallace RB, Wu L, Yood RA. Effect of hormone therapy on risk of hip and knee joint replacement in the Women's Health Initiative. Arthritis Rheum. 2006;54(10):3194-3120.

39. Thomas R, Williams M, Marshall C, Walker L. Switching to letrozole or exemestane improves hot flushes, mood and quality of life in tamoxifen intolerant women. Br J Cancer. 2008;98:1494-1499. 